\title{
Advanced Maternal Age and Severe Maternal Morbidity in South Korea: a Population-based Cohort Study
}

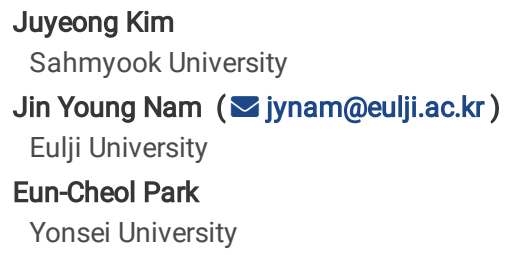

Research Article

Keywords:

Posted Date: February 9th, 2022

DOI: https://doi.org/10.21203/rs.3.rs-1275022/v1

License: () (i) This work is licensed under a Creative Commons Attribution 4.0 International License. Read Full License 


\section{Abstract}

Objective: We aimed to investigate the association between maternal age and severe maternal morbidity (SMM) in a Korean population.

Methods: Data for cases of delivery between 2003 and 2018 were extracted from the Korean National Health Insurance Service-National Delivery Cohort. The main outcome was SMM, which was determined using the Center for Disease Control and Prevention's algorithm. A generalized estimating equation model with a log link was performed for the relationship between SMM and maternal age-adjusted covariates.

Results: SMM occurred in 40,959/2,113,615 (1.9\%) of delivery cases. Teenagers and women 35 years and older had an increased risk of SMM in both nulliparous and multiparous cases (ages 15-19: adjusted risk ratio (RR) 1.32, 95\% confidence interval (Cl) 1.15-1.46; ages 35-39: RR 1.24, 95\% Cl 1.21-1.28; ages 40-44: RR 1.57, 95\% Cl 1.50-1.64; and ages 45 or older: RR 2.07, 95\% Cl 1.75-2.44).

Conclusion: Women aged 40 years and older had the highest rates of SMM. In singleton births as well as nulliparous and multiparous cases, teenagers and women ages 35 years and older had a particularly high risk of SMM. Identifying and managing risk factors for SMM in these vulnerable age groups may improve maternal health outcomes.

\section{Introduction}

Despite the overall decline in birth rate over the last decade, the number of births by women ages 30 years and older have increased ${ }^{1}$. From 2009 to 2019 , birth rates rapidly decreased by $13.4 \%$ for women aged $25-29$ years, $5.7 \%$ for women aged $30-34$ years, but increased for women aged 40 years and over in South Korea ${ }^{2}$. The socioeconomic environmental change of the development of assisted reproductive technology has caused women to have children at older ages. This trend of increasing births among older women is expected to last as reproductive technologies improve and become more accessible ${ }^{3}$.

Previously, older women were known to have underlying adverse maternal health outcomes due to their comorbid conditions such as diabetes, hypertension, and obesity. Even accounting for their existing diseases, healthy women with advanced maternal age have reported increasing rates of pregnancy complication ${ }^{4}$. Therefore, both prevention and management of adverse health outcomes after pregnancy for the advanced maternal age group is needed.

To manage adverse health outcomes after pregnancy, severe maternal morbidity (SMM) is a useful indicator for both improvement and assessment of maternal health services ${ }^{5}$. SMM includes both psychological and physical conditions that result from or worsen during pregnancy and may adversely influence a woman's health status. Women who develop SMM, compared to those who do not, experience increased medical costs, extended hospital stays, and potentially long-term adverse health outcomes ${ }^{6}$. Moreover, SMM can act as a substitute for risk factors for death by enabling faster identification and adjustment of potentially hazardous health systems and practice patterns ${ }^{7}$. Therefore, preventing and managing SMM by understanding the association between maternal age and SMM may be helpful in providing focused care for vulnerable populations after delivery discharge.

Previously, many studies exploring the association between maternal age and SMM have been conducted; however, the association between maternal age and SMM has been mixed. Some studies found a J-shaped association between maternal age and SMM, showing that the SMM of women of aged 45 years or older was the highest risk followed by women $40-44$ and women in teenage years, compared to those in their mid to late $20 \mathrm{~s}^{4,8-10}$. On the other hand, some studies found only a higher risk of SMM among women aged 35 years or older ${ }^{11-13}, 30$ years or older ${ }^{7,14}$, or only among women ages $30-39^{15}$. One study found higher SMM risk in women aged 35 years or older and lower SMM risk among women aged 18 years or younger ${ }^{16}$. Although previous studies have investigated the association between maternal age and SMM, the association remains unclear. In addition, many studies have been conducted on Western populations. Little information is available on the association between maternal age and SMM, especially in the Korean context.

Therefore, we performed a nationwide population-based cohort study of childbirth cases in South Korea. The purpose of this study was to investigate the association between maternal age and SMM. In addition, this study aimed to determine the association between age and the risk of SMM by parity and the status of multiple births.

\section{Methods \\ Data source}

This population-based cohort study was collected from the National Health Information Database (NHID) between 2013 and 2018 , which is composed of the health care utilization database, national health screening database, sociodemographic factors, and mortality for the entire South Korean population ${ }^{17}$. This data was formed by the National Health Insurance Service (NHIS), which is a single insurer covering the entire population of Korea ${ }^{17}$. The data linkage of the National Health Insurance could be made by using its unique resident registration numbers given to residents by the Korean government. The NHID uses deidentified join keys to substitute for personal identifiers to link these databases and to secure ethical clearance ${ }^{17}$. The healthcare utilization database, which is the biggest component of the NHID, is collected during the claim process of healthcare services, and includes information on records of inpatient and outpatient medical usage (diagnosis according to the International Classification of Diseases, 10th Revision (ICD-10), length of stay, treatment costs, and services received) and prescription records (drug code, days prescribed, daily dosage) ${ }^{17}$. The healthcare provider database contains the types of healthcare institutions, healthcare human resources, and equipment, covering all health care institutions in Korea ${ }^{17}$.

This study used the NHIS delivery cohort, which was extracted using the NHID claims database. The NHIS delivery cohort included all childbirth cases in South Korean healthcare institutions. This study defined childbirth using diagnosis and procedure codes for pregnancies with maternal age between ages 13 and 50 
who had a delivery hospitalization. The study population was included for at least 280 days before childbirth through 6 weeks following childbirth between January 1,2013 , and November 19,2018 . We defined childbirth as any record of inpatient hospitalization including a pregnancy-related diagnosis ${ }^{18}$ or procedure code for vaginal or cesarean delivery among pregnancies with more than 37 weeks of gestation. We excluded women who died during childbirth hospitalization, those who had more than 42 days of length of stay, and those who had no information on childbirth healthcare institutions. A total of 2,113,615 childbirths between 2013 and 2018 were included in this study.

\section{Ethics statement}

This research was approved by an ethical review from the Institutional Review Board of Eulji University in Korea (No. EU21-005), and all methods were performed in accordance with relevant guidelines and regulations. The requirement for informed consent was waived by the Institutional Review Board of Eulji University in Korea because all analysis used de-identified NHIS data.

\section{Severe maternal morbidity}

To determine SMM, we utilized the SMM algorithms developed by the Centers for Disease Control and Prevention (CDC) and included women who had at least one of the 21 previously established ICD-10 diagnosis and procedure codes during childbirth hospitalization ${ }^{18}$. The original SMM algorithm was 25 SMM indicators based on the 9 th revision of ICD ${ }^{6}$; however, in October 2015, the United States transitioned from the ICD-9 to ICD-10 diagnoses and procedures codes. The updated SMM algorithm represents serious complications of pregnancy or childbirth, such as eclampsia or acute renal failure, or procedures for the care of serious conditions, such as blood transfusion or hysterectomy. The 21 SMM indicators were composed of 16 diagnosis codes and five procedure codes.

\section{Maternal Age}

Maternal age at index childbirth was the main exposure variable of interest. Maternal age was divided into 5-year age groups and used as a categorical variable in this study.

\section{Covariates}

Confounders were selected based on previous studies as follows: household income level (quintile), type of insurance (self-employed insured, employee insured, and medical aid), and residential area (city and rural) were included as socioeconomic factors. Clinical factors included mode of delivery (spontaneous vaginal delivery, instrumental delivery, and cesarean section delivery), prenatal care (adequate, intermediate, or inadequate) using Kessner's Adequacy of Prenatal Care Index ${ }^{19}$, parity (nulliparous or multiparous), twin birth status (singleton and twin birth), and maternal comorbidities ( 0 , or 1 and more) using Howell's study ${ }^{20}$. Other covariates included the type of hospitals, which were divided by the number of beds (beds $<30,30 \leq$ beds $<100,100 \leq$ beds $<500$, and beds $\geq 500$ ), hospital regions (Seoul, other metropolitan areas, small cities, and rural areas), and the calendar year of childbirth.

\section{Statistical analysis}

We calculated the distribution of the general characteristics of the study population by maternal age at childbirth between 2013 and 2018 . The association between maternal age and SMM during childbirth hospitalization was determined using a Poisson regression model with a robust error variance adjusted for all covariates, which estimated the adjusted risk ratio (RR) and 95\% confidence intervals (CI). This repeated model was generated for stratified analysis of the relationship between mode of delivery, parity, or twin birth status and SMM by maternal age and evaluated for interactions. All statistical analyses were conducted using SAS (version 9.4; SAS Institute, Inc., Cary, NC, USA). The level of significance was set at $p<0.05$.

\section{Results}

Table 1 shows the general characteristics of the study population according to maternal age groups. Of the 2,113,615 women who had childbirth during the study period, 40,959 (1.9\%) episodes of SMM occurred during the childbirth hospitalization period. In terms of maternal age, women of ages 45 years or older had the highest incidence of SMM (5.3\%), and the 2nd and 3rd highest incidences of SMM were the teenage pregnancy group (3.4\%), and the 40 to 44 years old pregnancy group (3.3\%) 


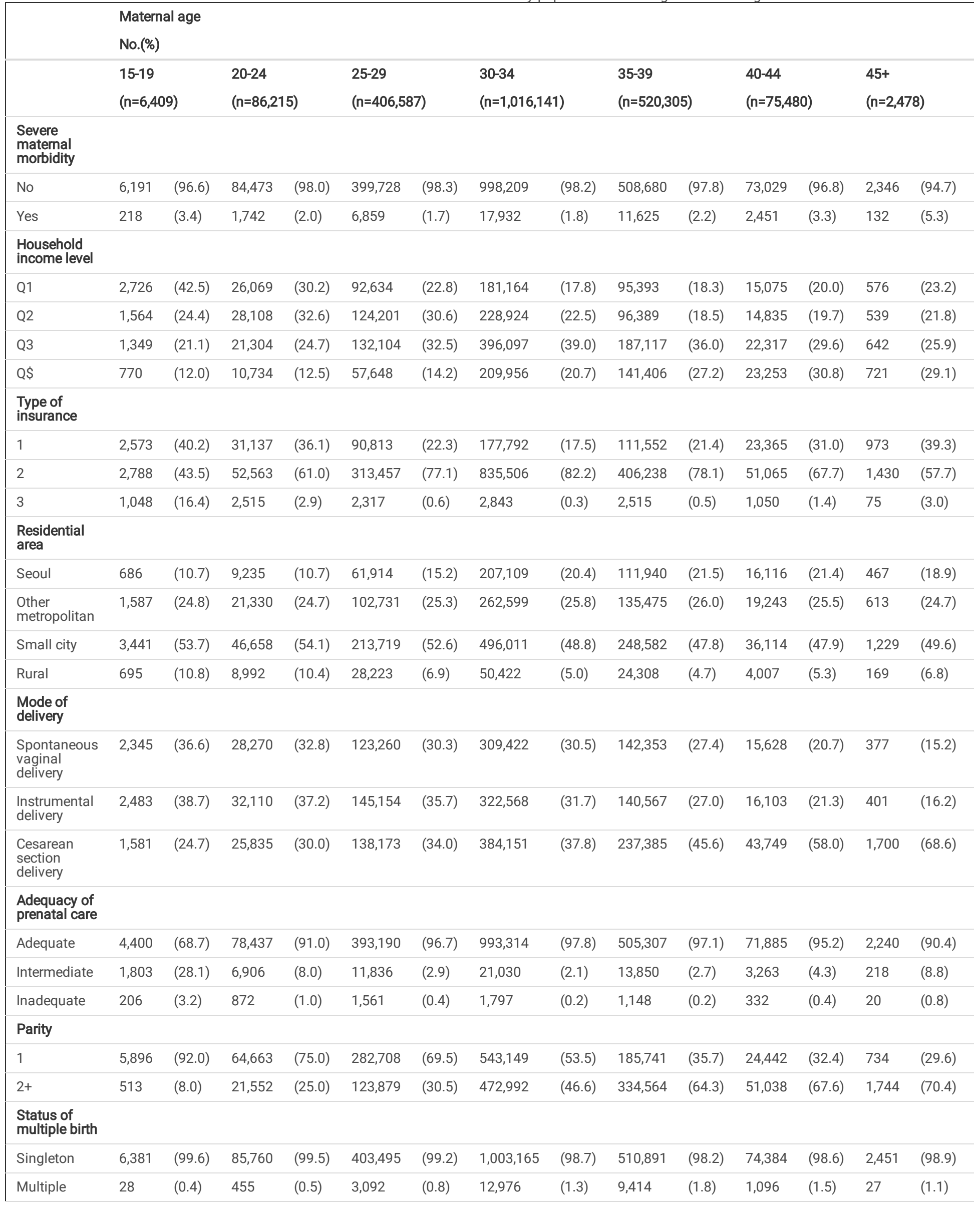




\begin{tabular}{|c|c|c|c|c|c|c|c|c|c|c|c|c|c|c|}
\hline \multirow[b]{2}{*}{$\begin{array}{l}\text { Maternal } \\
\text { comorbidity }\end{array}$} & \multicolumn{14}{|c|}{$\begin{array}{l}\text { Maternal age } \\
\text { No.(\%) }\end{array}$} \\
\hline & & & & & & & & & & & & & & \\
\hline 0 & 3,862 & $(60.3)$ & 48,834 & (56.6) & 218,633 & (53.8) & 416,105 & $(41.0)$ & 191,510 & (36.8) & 25,251 & (33.5) & 795 & (32.1) \\
\hline $1+$ & 2,547 & (39.7) & 37,381 & (43.4) & 187,954 & $(46.2)$ & 600,036 & (59.1) & 328,795 & (63.2) & 50,229 & (66.6) & 1,683 & (67.9) \\
\hline \multicolumn{15}{|l|}{$\begin{array}{l}\text { Type of } \\
\text { hospital (No. } \\
\text { of beds) }\end{array}$} \\
\hline $500+$ & 294 & $(4.6)$ & 2,029 & $(2.4)$ & 12,608 & (3.1) & 46,119 & (4.5) & 32,857 & (6.3) & 6,985 & $(9.3)$ & 377 & (15.2) \\
\hline $100-499$ & 617 & $(9.6)$ & 4,896 & $(5.7)$ & 22,240 & (5.5) & 67,274 & (6.6) & 45,781 & $(8.8)$ & 9,424 & (12.5) & 403 & (16.3) \\
\hline $30-99$ & 2,616 & $(40.8)$ & 39,386 & (45.7) & 200,893 & (49.4) & 512,092 & (50.4) & 254,096 & (48.8) & 33,698 & (44.6) & 985 & (39.8) \\
\hline$<30$ & 2,882 & $(45.0)$ & 39,904 & $(46.3)$ & 170,846 & $(42.0)$ & 390,656 & (38.5) & 187,571 & $(36.1)$ & 25,373 & (33.6) & 713 & (28.8) \\
\hline \multicolumn{15}{|l|}{$\begin{array}{l}\text { Hospital } \\
\text { region }\end{array}$} \\
\hline Seoul & 724 & (11.3) & 9,559 & (11.1) & 63,153 & (15.5) & 211,657 & (20.8) & 119,366 & (22.9) & 18,096 & (24.0) & 559 & (22.6) \\
\hline $\begin{array}{l}\text { Other } \\
\text { metropolitan }\end{array}$ & 1,984 & (31.0) & 24,656 & (28.6) & 117,562 & (28.9) & 294,779 & $(29.0)$ & 149,374 & (28.7) & 21,221 & $(28.1)$ & 707 & (28.5) \\
\hline Small city & 3,635 & (56.7) & 50,966 & $(59.1)$ & 223,964 & (55.1) & 506,620 & $(49.9)$ & 249,954 & $(48.0)$ & 35,824 & $(47.5)$ & 1,190 & (48.0) \\
\hline Rural & 66 & $(1.0)$ & 1,034 & $(1.2)$ & 1,908 & $(0.5)$ & 3,085 & $(0.3)$ & 1,611 & $(0.3)$ & 339 & $(0.5)$ & 22 & $(0.9)$ \\
\hline \multicolumn{15}{|l|}{ Year } \\
\hline 2013 & 1,494 & (23.3) & 17,533 & $(20.3)$ & 81,283 & $(20.0)$ & 204,243 & (20.1) & 78,816 & (15.2) & 12,091 & $(16.0)$ & 370 & (14.9) \\
\hline 2014 & 1,392 & (21.7) & 16,523 & (19.2) & 76,362 & (18.8) & 196,015 & (19.3) & 83,172 & $(16.0)$ & 12,171 & $(16.1)$ & 363 & (14.7) \\
\hline 2015 & 1,100 & $(17.2)$ & 16,156 & (18.7) & 74,285 & (18.3) & 190,326 & (18.7) & 92,315 & (17.7) & 12,763 & (16.9) & 388 & (15.7) \\
\hline 2016 & 1,039 & (16.2) & 14,589 & (16.9) & 67,990 & (16.7) & 168,352 & (16.6) & 94,125 & (18.1) & 12,953 & (17.2) & 457 & (18.4) \\
\hline 2017 & 796 & (12.4) & 12,076 & $(14.0)$ & 59,039 & (14.5) & 140,316 & (13.8) & 89,950 & (17.3) & 13,217 & (17.5) & 467 & (18.9) \\
\hline 2018 & 588 & (9.2) & 9,338 & (10.8) & 47,628 & (11.7) & 116,889 & (11.5) & 81,927 & (15.8) & 12,285 & (16.3) & 433 & (17.5) \\
\hline
\end{tabular}

Table 2 presents the association between maternal age and SMM, adjusted for all covariates. The risk of SMM by maternal age presented a J-shape based on the 25 to 29 age group. The maternal group aged 45 years and older had an approximately 2-fold higher risk of SMM than those aged 25 to 29 years (RR 2.17 , $95 \% \mathrm{Cl} 1.06-1.12)$. Women who were $40-44$ years old had an approximately 1.6 -fold higher risk of SMM (RR $1.57,95 \% \mathrm{Cl} 1.50-1.64)$, and those who were teenaged had an approximately 1.3-fold higher risk of SMM (RR 1.32, 95\% Cl 1.15-1.51) compared to the reference group. The risk of SMM was higher in women who had instrumental delivery and cesarean section delivery, in those who had inadequate prenatal care, those who were nulliparous, those who had twin births, those who had maternal comorbidities, and those who had childbirth in the tertiary hospital, compared to the reference group. 
Relative risk for the relationship between maternal age and SMM adjusted for all covariates.

\section{SMM}

RR

$(95 \% \mathrm{Cl})$

Matemal age (years)

$15-19$

20-24

25-29

30-34

35-39

40-44

$45+$

Household income level

Q1

Q2

Q3

Q4

Type of insurance

2

3

Residential area

Seoul

Other metropolitan

Small city

Rural

Mode of delivery

Spontaneous vaginal delivery

Instrumental delivery

Cesarean section delivery

\section{Adequacy of prenatal care}

Adequate

Intermediate

Inadequate

Parity

1

$2+$

Status of multiple birth

Singleton

Multiple

Matemal comorbidity

0

$1+$

Adjusted for household income level, type of insurance, residential area, mode of delivery, adequacy of prenatal care, parity, status of multiple birth, maternal comorbidity, type of hospital, hospital region, and year.
1.00

1.61

1.56

1.11

(1.08 -

1.13)

1.00

2.09

(1.92 -

2.28)

1.00

1.09

(1.04 -

1.15)

1.18

(1.14 -

1.22)

1.20

(1.14 -

1.27)

1.51)

1.18)

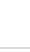

)

1.66)

(1.52 - 1.60)

1.00

1.45

(1.38 -

1.52)

1.54

(1.33 -

1.78)

1.34

(1.31 -

1.36)

1.00

2.04

(1.95 -

2.13)

1.00

1.00

1.31

(1.29 -

1.34)

Page 6/10 


\section{Type of hospital (No. of beds)}

\begin{tabular}{|c|c|c|c|}
\hline $500+$ & 4.33 & $(4.20-$ & 4.47) \\
\hline $100-499$ & 2.44 & $(2.36-$ & 2.51) \\
\hline $30-99$ & 1.00 & & \\
\hline$<30$ & 1.04 & $(1.02-$ & 1.07) \\
\hline \multicolumn{4}{|l|}{ Hospital region } \\
\hline Seoul & 1.00 & & \\
\hline Other metropolitan & 1.23 & $(1.18-$ & 1.29) \\
\hline Small city & 1.10 & $(1.06-$ & 1.14) \\
\hline Rural & 1.18 & $(1.01-$ & 1.38) \\
\hline \multicolumn{4}{|l|}{ Year } \\
\hline 2013 & 1.00 & & \\
\hline 2014 & 0.96 & $(0.93-$ & 0.99) \\
\hline 2015 & 1.02 & $(0.99-$ & 1.05) \\
\hline 2016 & 1.00 & $(0.97-$ & 1.04) \\
\hline 2017 & 0.95 & $(0.92-$ & $0.98)$ \\
\hline 2018 & 0.97 & $(0.94-$ & 1.00) \\
\hline
\end{tabular}

Adjusted for household income level, type of insurance, residential area, mode of delivery, adequacy of prenatal care, parity, status of multiple birth, maternal comorbidity, type of hospital, hospital region, and year.

We also performed a stratified analysis of parity and twin birth status on SMM by maternal age, which was confirmed as an interaction term. In parity, women ages 40 years and older had approximately 1.5 -folds higher risk of SMM in both nulliparous and multiparous women, compared to the reference group (nulliparous: 40-44 years: RR 1.47, 95\% Cl 1.38-1.59, 45+: RR 1.48, 95\% Cl 1.04-2.06; multiparous: $40-44$ years: RR 1.57, 95\% Cl 1.47-1.69; 45+: RR 2.18, 95\% Cl 1.79-2.64). In twin birth status, women ages 40 years and older had a 1.59- and 2.1-fold higher risk of SMM in singleton, respectively (40-44 years: RR 1.59, $95 \% \mathrm{Cl} 1.52-1.67 ; 45+$ RR $2.06,95 \% \mathrm{Cl} 1.74-2.44)$, but the only statistically significant association was between women aged 45 and older and the risk of SMM with multiple births (RR 2.39, 95\% Cl 1.11-5.13) (Table 3).

Table 3

The relationship between maternal age and severe maternal morbidity, stratified by mode of delivery, parity, and status of multiple birth.

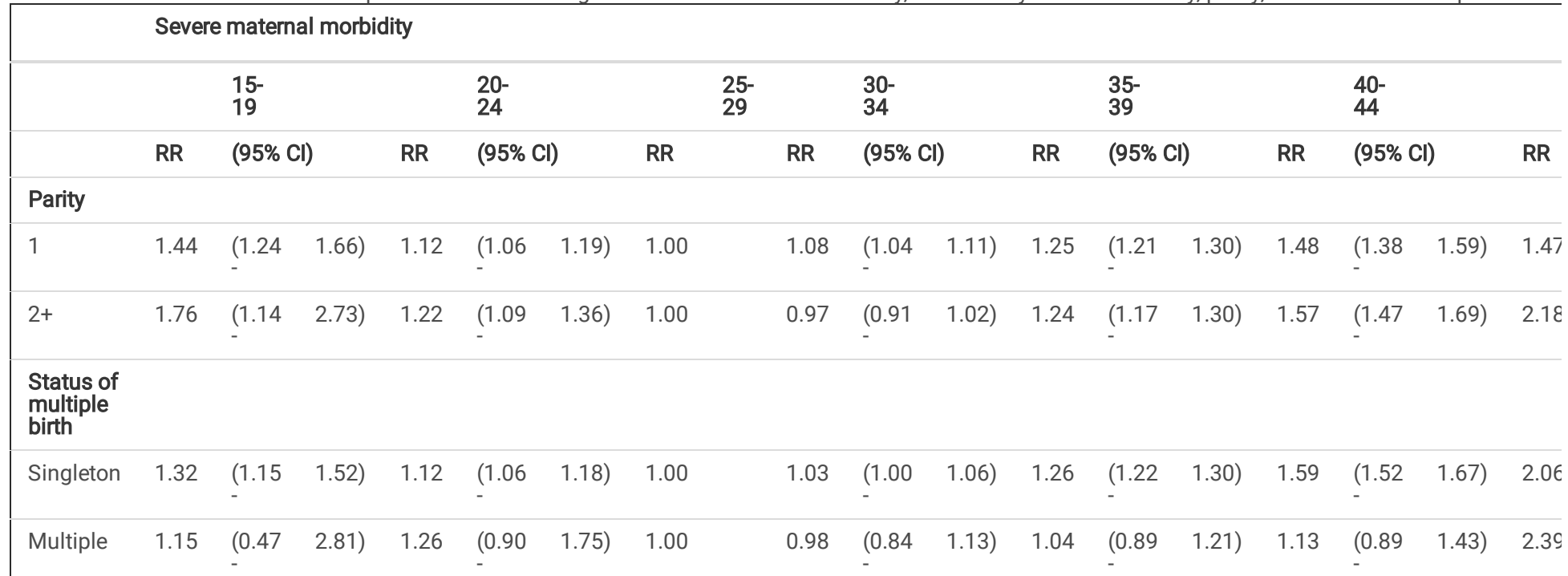

Adjusted for household income level, type of insurance, residential area, mode of delivery, adequacy of prenatal care, parity, status of multiple birth, maternal of hospital, hospital region, and year, except for the stratum itself.

\section{Discussion}


Using nationwide cohort data, this study examined the association between maternal age and SMM. We found that the risk curve of SMM by maternal age exhibits a J-shape, whereby younger and older women have the highest rates of SMM, with the maternal group of ages 45 years and older having the highest. Furthermore, women 45 years and older had a particularly high risk of SMM among nulliparous and multiparous women, and both singleton and twin births.

Previously, many studies regarding the association between maternal age and SMM have been performed, but the relationship between maternal age and SMM is unclear. The findings of this study are consistent with those of a previous study addressing a J-shaped relationship between maternal age and SMM

4,8-10. Considering the limited information about Asian studies regarding this issue, this study added evidence to investigate the association between maternal age and SMM based on data from a nationally representative Korean population.

The study findings can be explained as follows. First, the risk curve of SMM by maternal age exhibits a J-shape whereby younger and older women have higher rates of SMM, with maternal group aged 45 years and older having the highest. Baseline factors associated with aging, including hypertension, diabetes, or lower cardiac output, could make older mothers more vulnerable to physiological changes during pregnancy, thereby increasing the risk of SMM ${ }^{8}$. In addition, a previous study found that women of ages 15-17 were more likely to have eclampsia than any other study group ${ }^{4}$. Considering that eclampsia is one of the illnesses comprising SMM ${ }^{18}$, a higher SMM among women ages 15-19 than among women ages 25-29 years could be possible. Second, the stratified analysis of parity indicated that the risk of SMM was high in both 1 parity and multiparous women, and the risk of SMM was particularly high among multiparous women of ages 45 years and older, as compared to women with one parity. A previous study noted that pregnancy complications and adverse pregnancy outcomes were associated with increasing parity ${ }^{14,21}$, and this might be a factor that worsens SMM among older mothers. Finally, the stratified analysis of twin birth status indicated that the risk of SMM was high in both singleton and twin births, and the risk of SMM was particularly high among women ages 45 years and older. Previously, the risk of SMM during pregnancy and postpartum periods was found more frequently among women with twin births than among singleton pregnancies ${ }^{22}$. In addition, in those with a maternal age of 40 years and older, multiple births were found to be one of the strongest risk factors for SMM ${ }^{14}$. These factors may lead to the most adverse SMM.

South Korea has suffered from the problem of low fertility. The total fertility rate is 0.84 in 2020 , which is the lowest fertility rate among the Organisation for Economic Co-operation and Development (OECD) countries ${ }^{23}$. Numerous professionals have pointed out that later marriage and advanced maternal age have contributed to this problem. The average age of first marriage was 30.78 years old in 2020 , which is significantly increased compared to 26.49 years old in $2000^{24}$. The average age of first childbirth was 32.3 years old, which has increased 4.6 years since $2000^{25}$. These social phenomena affect advanced maternal age, which might also affect adverse maternal health outcomes, such as increasing the maternal mortality ratio or the risk of SMM. Therefore, it is necessary to monitor whether maternal age affects health outcomes and to make additional efforts to improve maternal health status.

This study had several strengths. To the best of our knowledge, this is the first study to examine the association between maternal age and SMM based on a nationally representative Korean delivery cohort. Furthermore, this study encompasses a very large population and long-term study because this database includes 18 years of follow up data for all Korean women who have delivered. Second, this study provides meaningful results in that not only nulliparous but also multiparous women can affect their risk of SMM by being of advanced maternal age, especially for women aged 40 years and older. This might be of value to countries which have late childbirth trends and may inform them how to avoid preventable maternal deaths or adverse maternal health outcomes. Third, this study considered several risk factors, including socioeconomic status, obstetric status, and provision factors. However, this study does have some limitations. First, the incidence of SMM might have been underestimated. Medical practitioners could under-report the incidence of SMM in medical records to report better quality indicators of services. In addition, the observational nature of our study leaves room for residual confounding and other potential sources of bias, such as maternal BMI, or alcohol or smoking habits. We could not control some risk factors that were not included in this database.

This study has several implications. As it has been confirmed that SMM is higher in teens and older women than in women in their late 20s, additional efforts to identify and manage risk factors for SMM in vulnerable age groups are needed. In addition, though it is classified as a risk group from the age of 35 years or older, the relatively young 35-39 year-old group was identified as a group with high SMM regardless of whether it was nulliparous or not. Considering these results, it would be necessary to highlight the importance of optimal childbirth age to prevent adverse maternal health outcomes in order to ensure a healthy life after childbirth

\section{Conclusion}

The risk of SMM by maternal age exhibits a J-shape whereby younger and older women have the highest rates of SMM, with maternal group ages 40 years and older having the highest. Furthermore, women age 40 years and older had a particularly high risk of SMM in nulliparous and multiparous women, as well as singleton births. To improve maternal health outcomes, policymakers need more specific efforts to identify and manage risk factors for SMM in these vulnerable age groups.

\section{Declarations}

\section{Data Availability}

The datasets generated during and/or analyzed during the current study are available from the corresponding author on a reasonable request.

\section{Acknowledgments}

This research was supported by the Basic Science Research Program through the National Research Foundation of Korea (NRF) funded by the Ministry of Science, ICT and Future Planning (No. 2020R1C1C1013668). 


\section{Author Contributions}

J.Y.N. performed statistical analyses. J.K. and J.Y.N. interpreted the data and drafted the manuscript. J.Y.N. designed and conducted the research and made substantial contributions to interpretation of data, and editing of the manuscript. E.C.P. and J.K. made substantial contributions to interpretation of data and revision of the manuscript. All authors revised manuscript for important intellectual content and gave final approval of the version to be published.

\section{Competing Interest Statement}

The authors declare no competing interests.

\section{References}

1. Shin Y-J. Results of Cohort Total Fertility Rates and Implications in Korea. In: Affairs KIfHaS, ed2019.

2. Statistics Korea. Statistics of Birth in 2019. 2020.

3. Hodes-Wertz B, Druckenmiller S, Smith M, Noyes N. What do reproductive-age women who undergo oocyte cryopreservation think about the process as a means to preserve fertility? Fertility and sterility. 2013;100(5):1343-1349. e1342.

4. Sheen J-J, Wright JD, Goffman D, et al. Maternal age and risk for adverse outcomes. American journal of obstetrics and gynecology. 2018;219(4):390. e391-390. e315.

5. Hill K, Thomas K, AbouZahr C, et al. Estimates of maternal mortality worldwide between 1990 and 2005: an assessment of available data. The Lancet. 2007;370(9595):1311-1319.

6. Callaghan WM, Creanga AA, Kuklina EV. Severe maternal morbidity among delivery and postpartum hospitalizations in the United States. Obstet Gynecol. 2012;120(5):1029-1036.

7. Lipkind H, Zuckerwise L, Turner E, et al. Severe maternal morbidity during delivery hospitalisation in a large international administrative database, 20082013: a retrospective cohort. BJOG: An International Journal of Obstetrics \& Gynaecology. 2019;126(10):1223-1230.

8. Aoyama K, Pinto R, Ray JG, et al. Association of maternal age with severe maternal morbidity and mortality in Canada. JAMA network open. 2019;2(8):e199875-e199875.

9. Frederiksen BN, Lillehoj CJ, Kane DJ, Goodman D, Rankin K. Evaluating lowa severe maternal morbidity trends and maternal risk factors: $2009-2014$. Maternal and child health journal. 2017;21(9):1834-1844.

10. Wen T, Overton EE, Sheen J-J, et al. Risk for postpartum readmissions and associated complications based on maternal age. The Journal of MaternalFetal \& Neonatal Medicine. 2021;34(9):1375-1381

11. Lisonkova S, Potts J, Muraca GM, et al. Maternal age and severe maternal morbidity: a population-based retrospective cohort study. PLoS medicine. 2017;14(5):e1002307.

12. Norhayati MN, Hazlina NHN, Aniza AA, Sulaiman Z. Factors associated with severe maternal morbidity in Kelantan, Malaysia: A comparative crosssectional study. BMC pregnancy and childbirth. 2016;16(1):1-10.

13. Oliveira FC, Surita FG, e Silva JLP, et al. Severe maternal morbidity and maternal near miss in the extremes of reproductive age: results from a national cross-sectional multicenter study. BMC pregnancy and childbirth. 2014;14(1):1-9.

14. Gray KE, Wallace ER, Nelson KR, Reed SD, Schiff MA. Population-based study of risk factors for severe maternal morbidity. Paediatric and perinatal epidemiology. 2012;26(6):506-514.

15. Stolk KH, Zwart JJ, Schutte J, Van Roosmalen J. Severe maternal morbidity and mortality from amniotic fluid embolism in the Netherlands. Acta obstetricia et gynecologica Scandinavica. 2012;91(8):991-995.

16. Moreira DdS, Gubert MB. Healthcare and sociodemographic conditions related to severe maternal morbidity in a state representative population, Federal District, Brazil: A cross-sectional study. PloS one. 2017;12(8):e0180849.

17. Cheol Seong S, Kim Y-Y, Khang Y-H, et al. Data resource profile: the national health information database of the National Health Insurance Service in South Korea. International journal of epidemiology. 2017;46(3):799-800.

18. National Center for Chronic Disease Prevention and Health Promotion. How Does CDC Identify Severe Maternal Morbidity? 2019; https://www.cdc.gov/reproductivehealth/maternalinfanthealth/smm/severe-morbidity-ICD.htm. Accessed April 20, 2021.

19. Kessner D SJ, Kalk C, Schlesinger E. . Infant Death: An Analysis by Maternal Risk and Health Care. Contrasts in Health Status. Vol. I. . Washington, DC: Institute of Medicine. National Academy of Sciences. ; 1973.

20. Howell EA, Zeitlin J, Hebert PL, Balbierz A, Egorova N. Association between hospital-level obstetric quality indicators and maternal and neonatal morbidity. Jama. 2014;312(15):1531-1541.

21. Aliyu MH, Jolly PE, Ehiri JE, Salihu HM. High parity and adverse birth outcomes: exploring the maze. Birth. 2005;32(1):45-59.

22. Madar H, Goffinet F, Seco A, Rozenberg P, Dupont C, Deneux-Tharaux C. Severe acute maternal morbidity in twin compared with singleton pregnancies. Obstetrics \& Gynecology. 2019;133(6):1141-1150.

23. Fertility rates (indicator). 2021. https://data.oecd.org/pop/fertility-rates.htm. Accessed 30 December 2021.

24. The age of the first marriage. Korea Statistics; 2021. https://kosis.kr/statHtml/statHtml.do? orgld=101\&tblld=DT_1B83A05\&vw_cd=MT_ZTITLE\&list_id=A23_2\&scrld=\&seqNo=\&lang_mode=ko\&obj_var_id=\&itm_id=\&conn_path=E1. Accessed December 31, 2021. 
25. The average maternal age of childbirth. Korea Statistics,; 2021. https://kosis.kr/statisticsList/statisticsListlndex.do? parentld=A.1\&vwcd=MT_ZTITLE\&menuld=M_01_01\#content-group. Accessed December 31, 2021. 